\title{
Food photography II: use of food photographs for estimating portion size and the nutrient content of meals
}

\author{
BY M. NELSON, M. ATKINSON AND S. DARBYSHIRE \\ Department of Nutrition and Dietetics, King's College London, Campden Hill Road, \\ London W8 7 AH
}

(Received 28 June 1994-Revised 13 September 1995 - Accepted 7 November 1995)

\begin{abstract}
The aim of the present study was to determine the errors in the conceptualization of portion size using photographs. Male and female volunteers aged 18-90 years $(n$ 136) from a wide variety of social and occupational backgrounds completed 602 assessments of portion size in relation to food photographs. Subjects served themselves between four and six foods at one meal (breakfast, lunch or dinner). Portion sizes were weighed by the investigators at the time of serving, and any waste was weighed at the end of the meal. Within $5 \mathrm{~min}$ of the end of the meal, subjects were shown photographs depicting each of the foods just consumed. For each food there were eight photographs showing portion sizes in equal increments from the 5 th to the 95 th centile of the distribution of portion weights observed in The Dietary and Nutritional Survey of British Adults (Gregory et al. 1990). Subjects were asked to indicate on a visual analogue scale the size of the portion consumed in relation to the eight photographs. The nutrient contents of meals were estimated from food composition tables. There were large variations in the estimation of portion sizes from photographs. Butter and margarine portion sizes tended to be substantially overestimated. In general, small portion sizes tended to be overestimated, and large portion sizes underestimated. Older subjects overestimated portion size more often than younger subjects. Excluding butter and margarine, the nutrient content of meals based on estimated portion sizes was on average within $\pm 7 \%$ of the nutrient content based on the amounts consumed, except for vitamin $C$ ( $21 \%$ overestimate), and for subjects over 65 years (15-20\% overestimate for energy and fat). In subjects whose BMI was less than $25 \mathrm{~kg} / \mathrm{m}^{2}$, the energy and fat contents of meals calculated from food composition tables and based on estimated portion size (excluding butter and margarine) were 5-10\% greater than the nutrient content calculated using actual portion size, but for those with BMI $30 \mathrm{~kg} / \mathrm{m}^{2}$ or over, the calculated energy and fat contents were underestimated by $2-5 \%$. The correlation of the nutrient content of meals based on actual or estimated portion sizes ranged from 0.84 to 0.96. For energy and eight nutrients, between 69 and $89 \%$ subjects were correctly classified into thirds of the distribution of intake using estimated portion size compared with intakes based on actual portion sizes. When 'average' portion sizes (the average weight of each of the foods which the subjects had served themselves) were used in place of the estimates based on photographs, the number of subjects correctly classified fell to between 60 and $79 \%$. We report for the first time the error associated with conceptualization and the nutrient content of meals when using photographs to estimate food portion size. We conclude that photographs depicting a range of portion sizes are a useful aid to the estimation of portion size. Misclassification of subjects according to their nutrient intake from one meal is reduced when photographs are used to estimate portion size, compared with the use of average portions. Age, sex, BMI and portion size are all potentially important confounders when estimating food consumption or nutrient intake using photographs.
\end{abstract}

Food photography: Survey methodology: Epidemiology

Minimizing measurement error is a key element in the successful elucidation of diet-disease relationships. In nutritional epidemiological studies, classification of subjects based on food consumption or nutrient intake will reflect the accuracy of the tool used to measure 
diet. There is no single measure of habitual diet in free-living subjects which is entirely valid. By using dietary assessment instruments for which the measurement errors have been described, the attenuating effects of subject misclassification on diet-disease relationships can be properly assessed and accounted for.

One of the main errors in the measurement of food consumption or nutrient intake occurs during the assessment of portion size. Where scales are used the problem is minimized, but there are many circumstances in which scales may not be available or when prospective assessment of diet is not appropriate. Assessment of the amounts of food consumed must then rely on subjects' ability to describe their consumption. Aids that have been used to help subjects to describe amounts include portion-size models (neutral shapes), replicate food models, and photographs. Photographs have the advantages of being easily copied, making them suitable for incorporation into questionnaires; they can include a wide range of individual foods, making them highly specific; and they can be posted, making them suitable for dietary assessments in large epidemiological studies.

A number of studies have reported the benefits of using photographs to help subjects assess portion size (Morgan et al. 1982; Rutishauser, 1982; Chu et al. 1984; Guthrie, 1984; Samet et al. 1984; Byers et al. 1985; Pietinen et al. 1988 a, b; Edington et al. 1989; Lee \& Cunningham, 1990; Hankin et al. 1991; Tjønneland et al. 1991; Faggiano et al. 1992). The number and size of photographs used to depict food portions have varied between studies, and there has been no systematic study of the ways in which photographs are interpreted for different foods or by different types of subjects. It is clear that the nature of the assessment will influence the outcome of the study (Gaskell et al. 1993). Not all studies report a positive outcome (Haraldsdottir et al. 1994).

For a subject the assessment of food portion sizes from photographs includes three main functions: perception (the ability to relate an amount of food which is present in reality to an amount depicted in a photograph); conceptualization (the ability to make a mental construct of an amount of food which is not present in reality, and to relate that to a photograph); and memory (which will affect the precision of the conceptualization). In a previous paper (Nelson et al. 1994) we addressed the issue of perception. Briefly, six foods were presented individually on a plate or in a bowl in varying amounts. Subjects were then asked (a) to identify the portion size using a visual analogue scale which related to eight photographs of the food ranging from the 5 th to the 95 th centile of portion weight based on The Dietary and Nutritional Survey of British Adults (British Adult Survey; Gregory et al. 1990), and (b) to estimate the portion on the plate as a fraction or multiple of the amount shown in a single photograph depicting an average (median) portion. Photographs were presented in different sizes and in black and white or in colour. Fifty-one subjects of both sexes ranging in age from 18-90 years and representing a wide spectrum of social and occupational backgrounds completed 7284 assessments. The mean average differences between the portion size presented on the plate and the estimate of the portion size based on photographs varied from $-4 \%$ to $+5 \%$ for the series of eight photographs, and from $-23 \%$ to $+9 \%$ for the single photograph. Large portion sizes tended to be underestimated. Subjects over 65 years tended to overestimate portion size more than younger subjects, and those with a $\mathrm{BMI} \geqslant 30 \mathrm{~kg} / \mathrm{m}^{2}$ tended to underestimate portion size in comparison with those with BMI $<30 \mathrm{~kg} / \mathrm{m}^{2}$. The general conclusions were (1) that the errors in the perception of portion size using photographs were generally small for a series of eight photographs; (2) that errors associated with the use of single (average) photographs were very much greater than for eight photographs; and (3) that elderly and overweight subjects may have consistently biased perceptions of food portion size in relation to others. This has important implications for differential misclassification in epidemiological studies embracing a wide cross-section of subjects. 
The present paper explores the role of conceptualization in the use of photographs to help subjects to estimate portion size. Through a systematic appraisal of assessments for a number of foodstuffs by subjects of both sexes from a wide age range and variety of backgrounds, the errors associated with conceptualization have been elucidated and quantified separately from those associated with perception alone.

\section{METHODS}

\section{Sample}

The aim was to obtain a good cross-section of men and women aged between 18 and 90 years from a wide variety of social and occupational backgrounds. Subjects were recruited through local employers, the local authority, voluntary organizations and day centres. Managers or organizers were asked to identify staff or members of their organization who might be willing to take part in the study. A letter and study information sheet prepared by the research team was given to each potential recruit. They were told that the purpose of the study was to assess the frequency with which foods are normally eaten, with no indication that portion size would be evaluated. Subjects were asked to respond individually in writing or by telephone to the name and address given in the letter (MA at King's College London). All subjects were volunteers, and none had participated in the earlier study. Table 1 shows the sample composition by age group and sex, giving mean height, weight, and BMI. Men aged 65 years and over were statistically significantly lighter (weight, $P=0.04$; BMI, $P=0.03$ ), than younger men. Women aged 65 years and over were significantly shorter than the younger women $(P=0.04)$.

\section{Choice of foods}

Twenty-two commonly eaten foods were chosen for testing. They excluded foods which are easy to describe in household measures (e.g. slices of bread, biscuits, eggs). The aim was to include foods for which some aid to portion size assessment would be necessary in an interview or questionnaire, and to span selected characteristics of appearance which were likely to influence perception of amounts from photographs: area and depth of pieces or mounds on a plate; number and size of pieces; area and thickness of slices; depth in a bowl, etc. The foods chosen and their appearance in the photographs are listed in Table 2. Butter and margarine were served with vegetables, bread and crackers, and baked beans were served either on their own or on toast. The subjects were shown the list of foods before recruitment and asked to confirm that they liked and would be willing to eat any of the foods on offer, in order to minimize the effect of plate waste and food preference as factors influencing conceptualization.

\section{Procedures}

Subjects came to the Nutrition Department at King's College London for one meal (breakfast, lunch or dinner). The foods served in each meal are listed in the Appendix. Between one and ten subjects were served a meal at one sitting. Menus were constructed and subjects invited for meals so as to diversify the range of subjects (age, sex, social class, etc.) consuming each food. Subjects served themselves all of the foods on offer for a given meal. As each food was served, it was weighed directly on the plate or bowl using a set of Salter digital food weighing scales $(3000 \mathrm{~g} \times 1 \mathrm{~g}$ ) (Salter Weigh-Tronix, West Bromwich, West Midlands), and the weight recorded by MA. If any foods were left over, the weight was recorded, and the actual amount eaten was calculated. In practice, the amounts left over were negligible.

Within 5 min of the completion of the meal, subjects were given a visual analogue scale (VAS) and a set of eight colour photographs for each of the foods they had eaten. The VAS 
Table 1. Mean height, weight and BMI in 136 subjects according to age group and sex

(Mean values and standard deviations)

\begin{tabular}{|c|c|c|c|c|c|c|c|}
\hline \multirow{2}{*}{$\begin{array}{l}\text { Age group } \\
\text { (years) }\end{array}$} & \multirow[b]{2}{*}{$n$} & \multicolumn{2}{|c|}{ Height (m) } & \multicolumn{2}{|c|}{ Weight (kg) } & \multicolumn{2}{|c|}{ BMI $\left(\mathrm{kg} / \mathrm{m}^{2}\right)$} \\
\hline & & Mean & SD & Mean & SD & Mean & SD \\
\hline \multicolumn{8}{|l|}{ Males } \\
\hline$<30$ & 22 & $1 \cdot 79$ & 0.08 & $80 \cdot 7$ & $14 \cdot 3$ & $25 \cdot 2$ & $3 \cdot 7$ \\
\hline $30-44$ & 22 & $1 \cdot 78$ & 0.07 & $88 \cdot 1$ & $17 \cdot 4$ & $28 \cdot 0$ & $5 \cdot 3$ \\
\hline $45-64$ & 20 & $1 \cdot 75$ & 0.08 & 86.7 & $10 \cdot 3$ & $28 \cdot 3$ & $2 \cdot 5$ \\
\hline $65+$ & 10 & $1 \cdot 75$ & 0.10 & $73 \cdot 1$ & $14 \cdot 5$ & $24 \cdot 4$ & $3 \cdot 7$ \\
\hline All ages & & $1 \cdot 77$ & 0.08 & $83 \cdot 6$ & $15 \cdot 0$ & $26 \cdot 8$ & $4 \cdot 2$ \\
\hline$P$ (ANOVA) & \multicolumn{3}{|c|}{0.40} & \multicolumn{2}{|c|}{0.04} & \multicolumn{2}{|c|}{0.03} \\
\hline \multicolumn{8}{|l|}{ Females } \\
\hline$<30$ & 23 & 1.63 & 0.06 & $62 \cdot 7$ & $11 \cdot 5$ & 23.6 & $4 \cdot 3$ \\
\hline $30-44$ & 15 & 1.65 & 0.07 & $68 \cdot 1$ & $10 \cdot 8$ & $25 \cdot 0$ & $3 \cdot 5$ \\
\hline $45-64$ & 13 & 1.62 & 0.06 & $64 \cdot 0$ & $8 \cdot 6$ & $24 \cdot 2$ & $2 \cdot 1$ \\
\hline $65+$ & 11 & 1.58 & 0.05 & 63.0 & $11 \cdot 2$ & $25 \cdot 3$ & $4 \cdot 6$ \\
\hline All ages & & 1.62 & 0.06 & $64 \cdot 4$ & $10 \cdot 5$ & $24 \cdot 4$ & $3 \cdot 8$ \\
\hline$P$ (ANOVA) & \multicolumn{3}{|c|}{0.04} & 0.4 & & \multicolumn{2}{|c|}{0.58} \\
\hline
\end{tabular}

was $11 \mathrm{~cm}$ long, and was marked with eight lines and numbers indicating the eight photographs, as shown below:

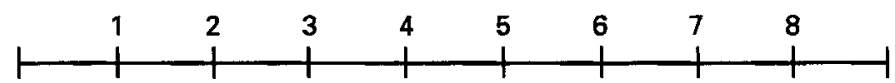

Each of the eight photographs in each set was A7 in size (approximately $75 \times 100 \mathrm{~mm}$, landscape) and showed one portion of food on a plate or in a bowl with either a knife and fork or dessertspoon (respectively) in view, photographed against a plain white background. The smallest and largest portion sizes depicted in the photographs represented the 5 th and 95th centiles of consumption for that food as recorded in the British Adult Survey (Gregory et al. 1990). The six remaining photographs depicted portion sizes at equal weight intervals between the smallest and the largest portion sizes. For each food, subjects were asked to mark a cross on the VAS which they believed most closely represented the size of the portion which they had consumed, allowing for any waste or food left over from the original serving. The six sets of photographs from stage 1 (Nelson et al. 1994) were also used in stage 2 .

For each food, 'average' portion size was calculated as the average weight of the food eaten by those subjects who had served themselves that food. The 'average' portion sizes were used in place of the estimates based on photographs to assess the effect of the practice of using an average portion size (e.g. in questionnaire analysis) to estimate nutrient intake.

For a sub-study on descriptors of milk portion sizes, twenty-one subjects who ate breakfast which included milk on cornflakes were asked to state in four ways the amount of milk added to the cereal: (1) number of tablespoons; (2) fraction of a pint; (3) 'small', 'medium' or 'large' portion; and (4) 'damp', 'normal' or 'drowned'.

The nutrient content of the meals was calculated from food consumption tables (Tan $e t$ al. 1985; Holland et al. 1988, 1989, 1991 a, b, 1992a,b). The nutrient content reported reflects the weights of all foods consumed at each meal, and each meal contained only those foods listed in the menus in the Appendix.

The statistical significance of differences between groups (Tables 3, 5 and 9) were assessed using two- or three-factor ANOVA, controlling for factors listed in the footnote in each 
FOOD PHOTOGRAPHY IN DIETARY SURVEYS




Table. Portion-size groups were defined according to the weights in the photographs: 'small' corresponded to weights less than that in photograph 3 ; 'medium' to weights between photograph 3 and photograph 6; and 'large' to weights greater than in photograph 6.

Ethical permission was granted by the King's College Research Ethics Committee.

\section{RESULTS}

Table 2 shows the mean difference in weight $(\mathrm{g})$ and the mean percentage difference in weight between the food consumed and the estimated portion size, together with the number of subjects consuming each food, and the age distribution of subjects. The size of the error differed markedly between foods, and in general was related to the average serving size and the amount consumed. The percentage errors ranged from underestimates of $28 \%$ (for baked beans) to overestimates of $242 \%$ (for butter or margarine on crackers). Over all foods there was an overestimate in portion size of about $32 \%$, but this fell to about $11 \%$ when butter and margarine were excluded. The correlation coefficients relating estimated portion weight to actual amount served ranged from 0.11 (for butter or margarine spread on crackers) to 0.97 for beef stew. The correlation coefficients for butter and margarine spread on bread and on crackers failed to reach statistical significance (i.e. were not different from zero). Regression of estimated $v$. actual weights yielded coefficients all below 1.0 (with the exception of shepherd's pie), indicating a consistent 'flat-slope' syndrome and 'regression to the mean' effect. The cumulative percentage of portion sizes estimated to within a given percentage of the weight served is shown in Fig. 1 for all foods, and excluding butter and margarine. Approximately $55 \%$ of foods ( $65 \%$ excluding butter and margarine) were estimated to within $\pm 30 \%$ of actual portion size.

Table 3 shows the effects of portion size, age, and BMI on over- or underestimation of portion size (as a percentage of the actual portion size), by sex. All analyses were carried out with and without butter and margarine, as the error associated with these foods was much greater than for the other foods. Significance levels given at the bottom of Table 3 relate to three-factor ANOVA, with portion size, age group, and BMI as main factors.

Generally, men tended to overestimate portion size more than females, particularly for small portion sizes. The differences in error between portion sizes were statistically significant (ANOVA, men $P<0.001$; women, $P=0.004$ (excluding butter and margarine)). The 'regression to the mean' effect was apparent, in that small portion sizes tended to be overestimated, and large portion sizes underestimated. Excluding butter and margarine, approximately $75 \%$ of medium and large portion sizes were estimated to within $\pm 30 \%$ of actual weight, but only $50 \%$ of estimates were accurate to within $\pm 30 \%$ for small portion sizes.

On average, men and women over the age of 65 years tended to overestimate portion size more than younger subjects, although the differences by age group reached statistical significance only for males ( $P=0.006$, excluding butter and margarine). There were no consistent trends in errors according to BMI.

Table 4 shows the average over- or underestimation of the energy and nutrient contents of meals based on estimates of portion size using photographs. Results are expressed as a percentage of the energy and nutrient content based on actual portion size, either for all foods in the meal or excluding butter and margarine. The nutrient content of the meals relates only to foods contained in the menus listed in the Appendix (no other foods were eaten at the meal), but it excludes the contribution from milk, as milk was not included in the estimates of portion size using photographs.

In line with the general overestimate of portion size, nutrients also tended to be overestimated. The extent of the overestimation varied from nutrient to nutrient, and was 


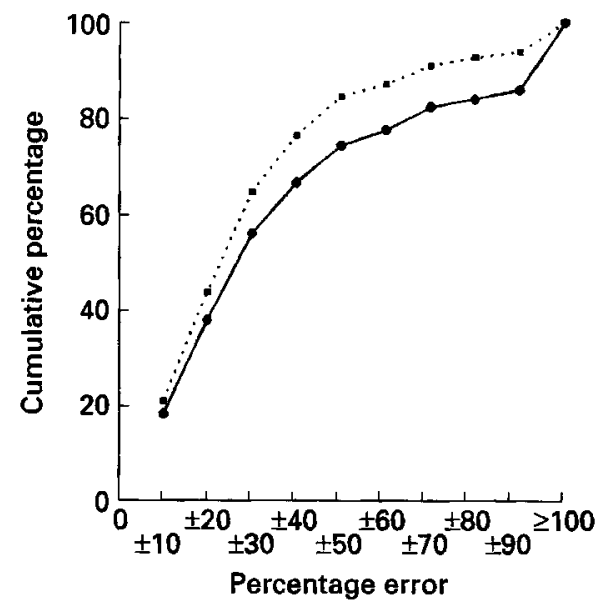

Fig. 1. Cumulative percentages of percentage errors between actual and estimated portion weights, with (O) or without $(\square)$ butter and margarine, for all foods. For details of procedures, see pp. 33-36.

most pronounced for fat and fatty acids when butter and margarine were included. When butter and margarine were excluded from the calculations, estimates were on average within $\pm 7 \%$ of the values based on the weighed foods, with the exception of vitamin C. The cumulative percentages of subjects with a given percentage error in the estimate of nutrient content of a meal (calculated from portion sizes using photographs $v$. actual weights of foods) is shown in Fig. 2 for energy and five nutrients. Of the estimates of the energy content of meals based on the use of photographs, $80 \%$ were within $\pm 25 \%$ of the energy content based on actual portion size. In contrast, less than $60 \%$ of the estimates of vitamin $C$ based on photographs were within $\pm 25 \%$ of the value based on actual portion size. Cumulative percentages for the other nutrients were intermediate between those for energy and vitamin $C$.

In Table 5 the effects of age and BMI on the estimates of energy and fat content of the meals are shown. Even when butter and margarine were excluded, the energy and fat contents of meals were significantly overestimated in the oldest group (controlling for sex and BMI in an ANOVA). This is reflected in the greater number of larger errors amongst the elderly: only $70 \%$ of calculated energy contents of meals based on estimated portion sizes were within $\pm 25 \%$ of the energy content based on actual portion sizes, compared with $80-85 \%$ correct to within $\pm 25 \%$ amongst the younger age groups. The influence of a high BMI ( $\left.\geqslant 30 \mathrm{~kg} / \mathrm{m}^{2}\right)$ on underestimation (when butter and margarine were excluded) was of borderline significance for energy $(P=0.069)$ but reached statistical significance for fat $(P=0.046)$, controlling for sex and age in the ANOVA. Errors in the estimated energy and fat content of meals were about 10 percentage points lower than in the lightest group $\left(\right.$ BMI $\left.<25 \mathrm{~kg} / \mathrm{m}^{2}\right)$.

Table 6 shows the extent to which subjects were correctly classified by thirds according to the energy and nutrient contents of meals based on actual or estimated portion size. The proportion correctly classified ranged from 69 to $89 \%$ when using all foods, and from 68 to $86 \%$ when butter and margarine were excluded. The most marked effect on classification when butter and margarine were excluded was the improvement in the classification for intakes of total fat ( $9 \%$ more subjects correctly classified) and saturated fatty acids ( $8 \%$ more subjects correctly classified). Correlations between the energy and nutrient content of meals based on actual or estimated portion sizes ranged from 0.84 (for Fe and NSP) to 0.96 (for polyunsaturated fatty acids). There were slight increases in the correlation coefficients 


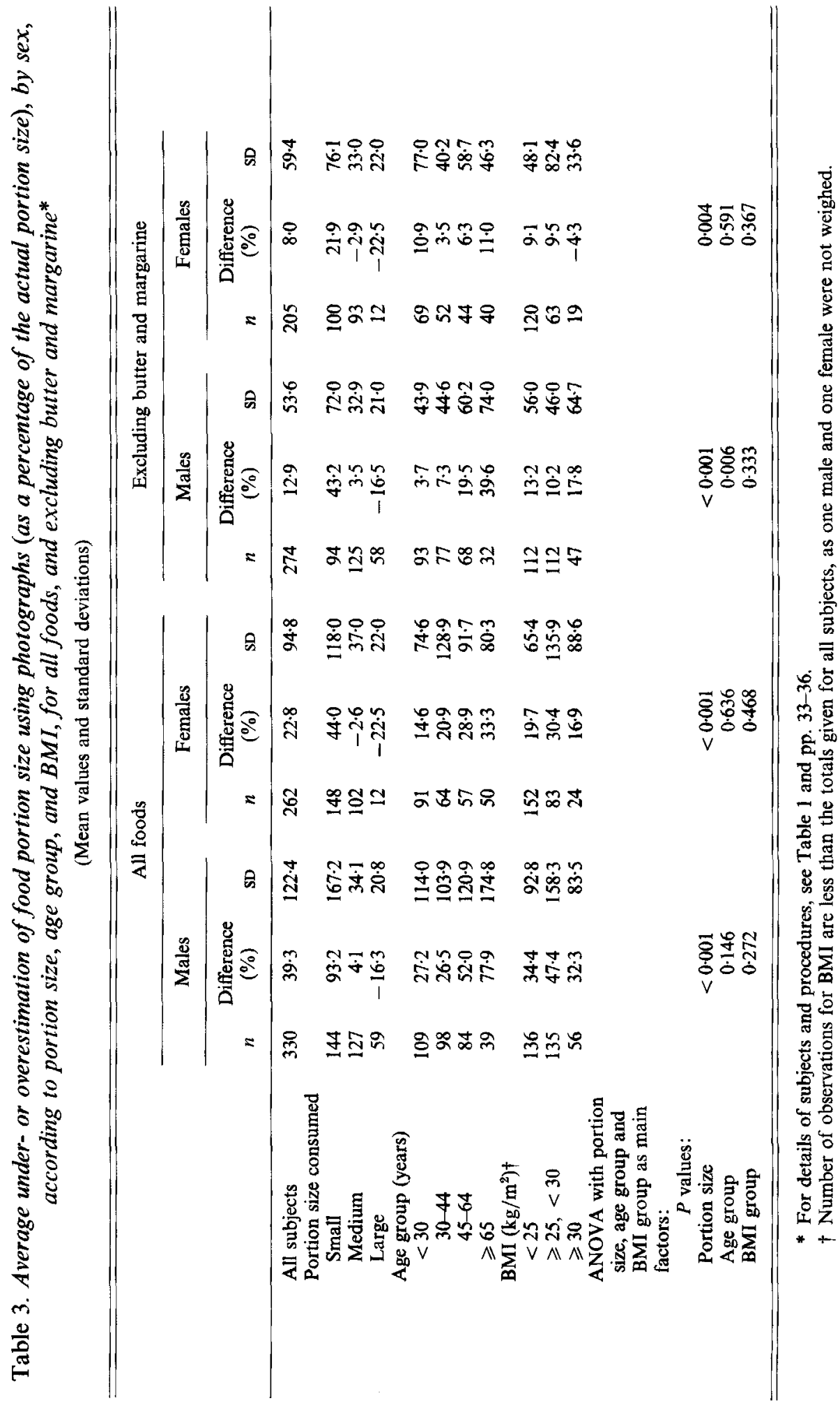


Table 4. Average under-or overestimation of the energy and nutrient contents of meals based on estimates of portion size using photographs (as a percentage of the nutrient content based on actual portion size), for all foods, and excluding butter and margarine, for 135 subjects* (Mean values and standard deviations)

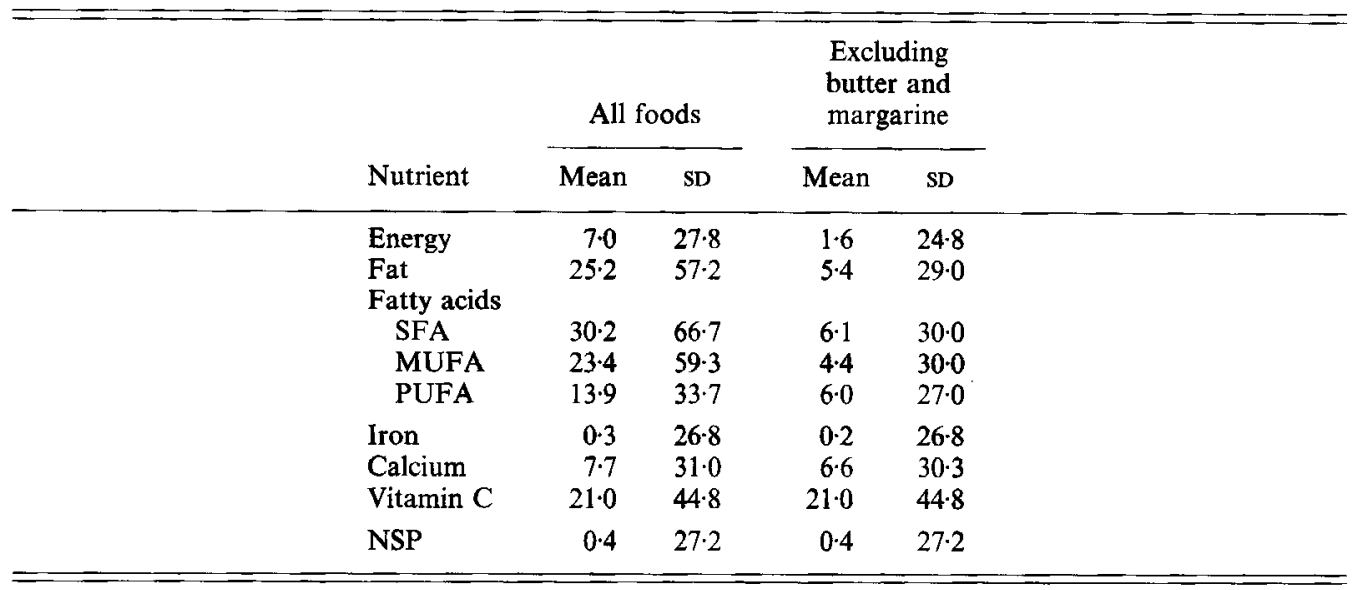

SFA, saturated fatty acids; MUFA, monounsaturated fatty acids; PUFA, polyunsaturated fatty acids.

* For details of subjects and procedures, see Table 1 and pp. 33--36.

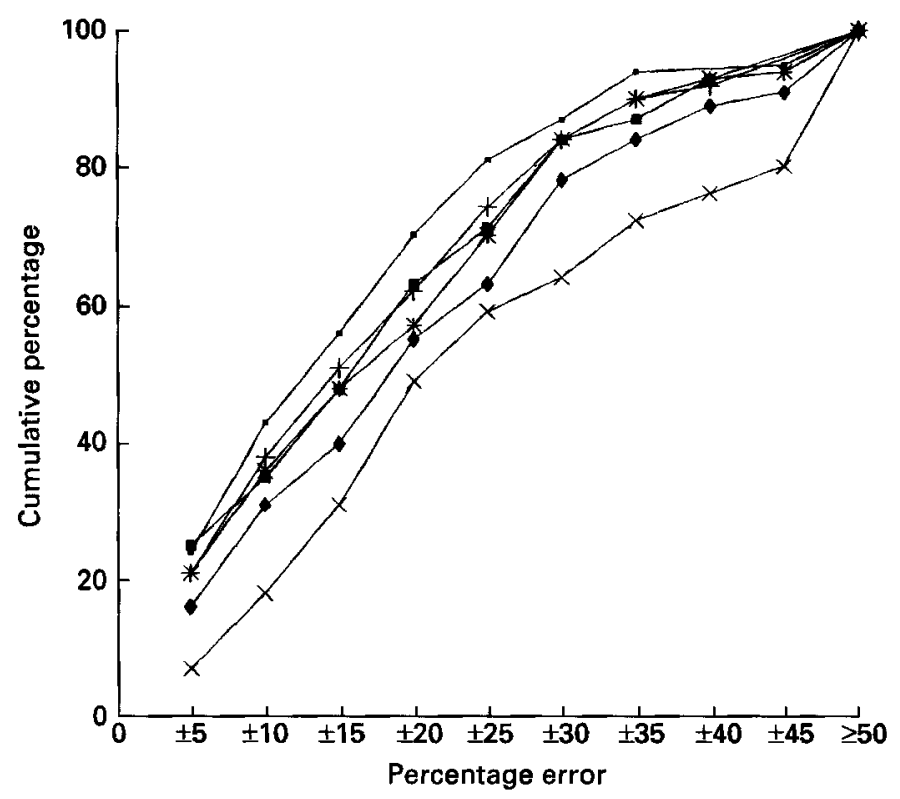

Fig. 2. Cumulative percentages of percentage errors in the nutrient content of meals based on estimates of portion size using photographs $v$. actual weights of foods, excluding butter and margarine. For details of procedures, see pp. 33-36. (a), Energy; (+), fat; (*), iron; ( $($ ), calcium; $(\times)$, vitamin C; $(\bullet)$, NSP.

for energy, total fat, saturated fatty acids and monounsaturated fatty acids when butter and margarine were excluded.

The effect of using an average portion size rather than photographs when classifying subjects according to the nutrient content of meals (excluding butter and margarine) is 
Table 5. Average under-or overestimation of the energy and fat contents of meals based on estimates of portion size using photographs (as a percentage of nutrient content based on actual portion size), according to age and BMI, for all foods, and excluding butter and margarine, for 135 subjects*

(Mean values and standard deviations)

\begin{tabular}{|c|c|c|c|c|c|c|c|c|c|}
\hline & \multirow[b]{3}{*}{$n$} & \multicolumn{4}{|c|}{ All foods } & \multicolumn{4}{|c|}{$\begin{array}{l}\text { Excluding butter and } \\
\text { margarine }\end{array}$} \\
\hline & & \multicolumn{2}{|c|}{ Energy } & \multicolumn{2}{|c|}{ Fat } & \multicolumn{2}{|c|}{ Energy } & \multicolumn{2}{|c|}{ Fat } \\
\hline & & Mean & SD & Mean & SD & Mean & SD & Mean & SD \\
\hline \multicolumn{10}{|c|}{ Age group (years) } \\
\hline$<30$ & 45 & -0.8 & $20 \cdot 5$ & $9 \cdot 7$ & $30 \cdot 4$ & $-3 \cdot 8$ & $16 \cdot 9$ & $-1 \cdot 0$ & $17 \cdot 7$ \\
\hline $30-44$ & 37 & $3 \cdot 9$ & $21 \cdot 4$ & $13 \cdot 8$ & $31 \cdot 7$ & $-1 \cdot 2$ & $18 \cdot 7$ & $8 \cdot 3$ & $24 \cdot 6$ \\
\hline $45-64$ & 33 & $11 \cdot 3$ & $29 \cdot 4$ & 51.5 & $90-4$ & $1 \cdot 1$ & $22 \cdot 7$ & $2 \cdot 3$ & $26 \cdot 1$ \\
\hline$\geqslant 65$ & 20 & 21.6 & $42 \cdot 1$ & $37 \cdot 6$ & $55-4$ & $15 \cdot 6$ & $43 \cdot 4$ & $19 \cdot 7$ & $50-7$ \\
\hline $\begin{array}{l}\text { ANOVA cont } \\
\text { for sex and }\end{array}$ & & & & & & & & & \\
\hline BMI $: P=$ & & \multicolumn{2}{|c|}{0.012} & \multicolumn{2}{|c|}{0.007} & \multicolumn{2}{|c|}{0.016} & \multicolumn{2}{|c|}{0.026} \\
\hline \multicolumn{10}{|l|}{ BMI $\left(\mathrm{kg} / \mathrm{m}^{2}\right)$} \\
\hline$<25$ & 66 & $9 \cdot 4$ & $31 \cdot 0$ & $21 \cdot 3$ & $41 \cdot 4$ & $5 \cdot 5$ & $29 \cdot 8$ & $10 \cdot 3$ & $33 \cdot 8$ \\
\hline$\geqslant 25,<30$ & 49 & 6.0 & $27 \cdot 0$ & $28 \cdot 8$ & $74 \cdot 3$ & $-1 \cdot 2$ & $18 \cdot 4$ & 0.8 & $23 \cdot 3$ \\
\hline & 18 & 0.5 & $16 \cdot 7$ & $30 \cdot 2$ & $59 \cdot 3$ & $-5 \cdot 3$ & $19 \cdot 0$ & -1.6 & \\
\hline \multicolumn{10}{|c|}{ ANOVA controlling } \\
\hline age: $P=$ & \multicolumn{3}{|c|}{0.289} & \multicolumn{2}{|c|}{0.870} & \multicolumn{2}{|c|}{0.069} & \multicolumn{2}{|c|}{0.046} \\
\hline
\end{tabular}

* For details of subjects and procedures, see Table 1 and pp. 33-36.

shown in the last four columns in Table 6 . Between 6 and $21 \%$ fewer subjects were correctly classified, and between 1 and $4 \%$ more subjects were grossly misclassified in the opposite third when using average portion sizes rather than estimates based on photographs. The correlation coefficients between the energy and nutrient contents of meals based on actual $v$. average portion sizes ranged from 0.74 (for vitamin $C$ ) to 0.9 (for polyunsaturated fatty acids), substantially lower than the correlations between the energy and nutrient contents of meals based on actual portion size $v$. estimates made using photographs. When standard portions based on the British Adult Survey were used, misclassification by thirds was greater still, and the correlation coefficients lower.

Table 7 shows the comparison between errors for the six foods used in stage 1 (perception of food portion size from photographs; Nelson et al. 1994) and the same foods in stage 2 (conceptualization of food portion size from photographs; the present study), based on the same sets of photographs. The average overestimate of portion size for these six foods increased slightly, from $0.8 \%$ in stage 1 to $4 \%$ in stage 2 . The differences were statistically significant for boiled potato, quiche, and sliced meat, but these became non-significant in an ANOVA controlling for age. The spread of values (as assessed by standard deviations) was similar for mashed and boiled potatoes, quiche, and spaghetti in both stages, but was substantially larger in stage 2 for cornflakes and sliced meat. The lower part of the table shows the cumulative percentage of observations correct to within the given percentage error. With the exception of quiche, a slightly smaller proportion of subjects in stage 2 than in stage 1 were successful in correctly identifying portion size at almost every level of error, although the differences were statistically significant only for boiled potato (KolmogorovSmirnov two-sample test, $P=0$-012). 
FOOD PHOTOGRAPHY IN DIETARY SURVEYS

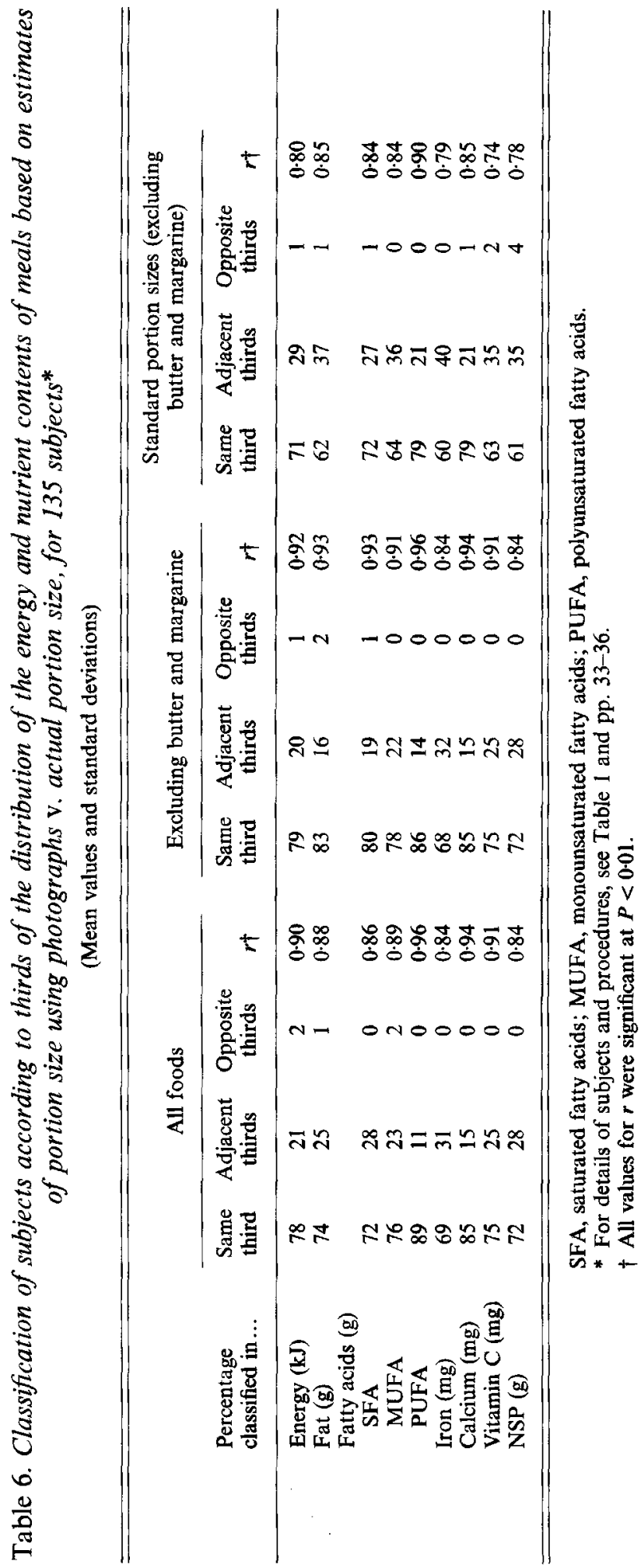




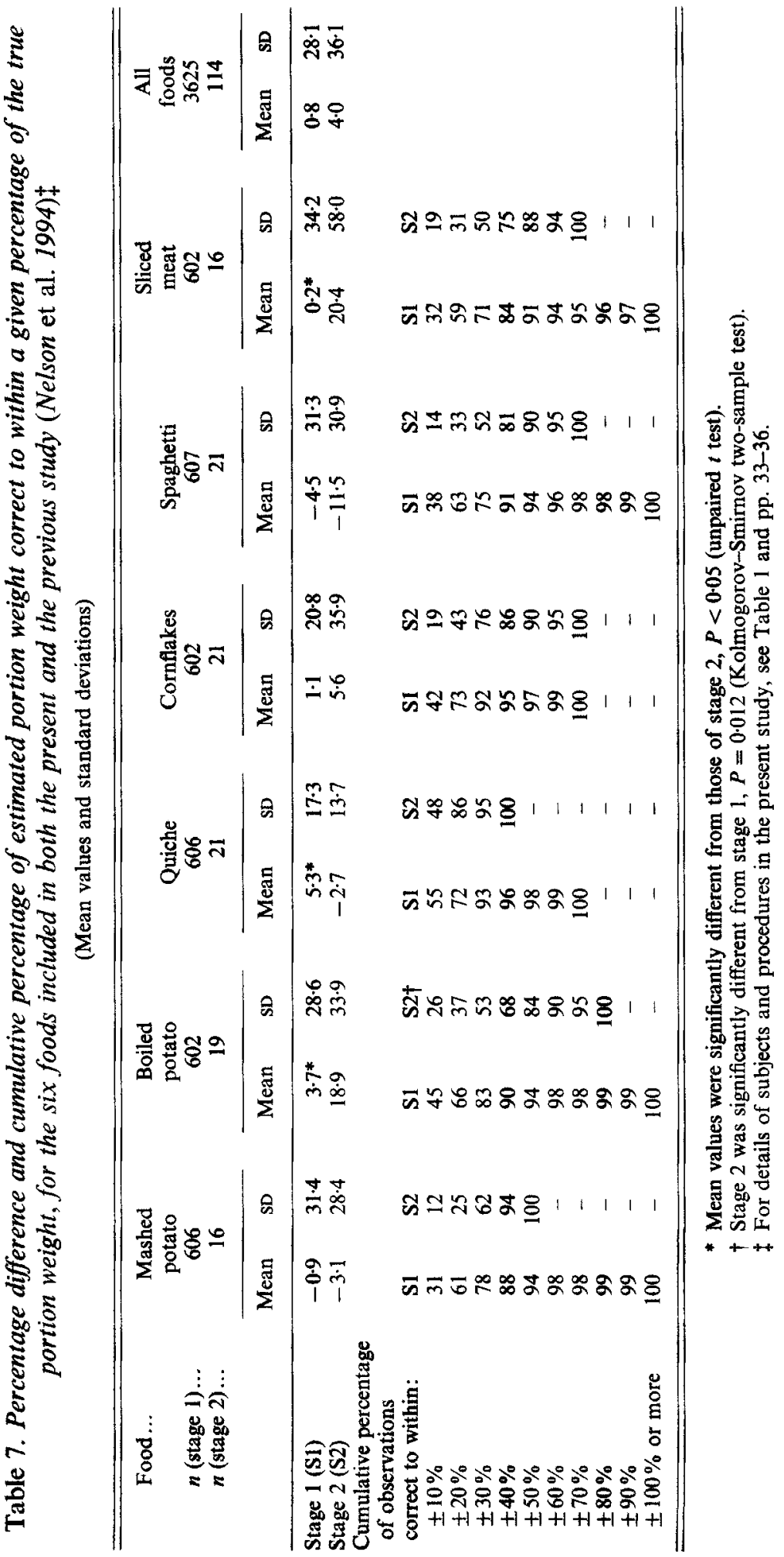


Table 8. Milk portion size $(g)$ in relation to various descriptors, for twenty-one subjects

(Values in parentheses show the number of portions described according to each measure (not all subjects provided information on all measures))

\begin{tabular}{|c|c|c|c|c|c|}
\hline \multicolumn{6}{|c|}{ Quantitative } \\
\hline $\begin{array}{l}\text { Number of } \\
\text { tablespoons } \dagger\end{array}$ & $\begin{array}{l}\text { Amount } \\
\text { served } \\
\text { (g) }\end{array}$ & $\begin{array}{l}\text { Fraction } \\
\text { of pint }\end{array}$ & $\begin{array}{l}\text { Amount } \\
\text { served } \\
\text { (g) }\end{array}$ & $\begin{array}{l}\text { Portion } \\
\text { of bowl }\end{array}$ & $\begin{array}{l}\text { Amount } \\
\text { served } \\
\text { (g) }\end{array}$ \\
\hline 2 & $23(1)$ & 0.1 & $62(9)$ & 0.1 & $46(4)$ \\
\hline 3 & $107(2)$ & 0.2 & $107(3)$ & 0.25 & $84(2)$ \\
\hline 4 & $71(1)$ & $0 \cdot 3$ & $104(6)$ & 0.33 & $77(2)$ \\
\hline 5 & $57(1)$ & 0.4 & - & 0.5 & $125(5)$ \\
\hline 6 & $130(1)$ & 0.5 & $164(1)$ & 0.75 & $130(1)$ \\
\hline 7 & $110(4)$ & & & & \\
\hline 8 & $75(5)$ & & & & \\
\hline 9 & $75(1)$ & & & & \\
\hline $10+$ & $115(2)$ & & & & \\
\hline \multicolumn{6}{|c|}{ Qualitative } \\
\hline Small & $74(8)$ & & Damp & $75 \quad(7)$ & \\
\hline Medium & $94(9)$ & & Normal & $87(11)$ & \\
\hline Large & $147(2)$ & & Drowned & 147 (2) & \\
\hline
\end{tabular}

* For details of procedures, see p. 34.

$\uparrow$ One tablespoon measure weighs $15 \mathrm{~g}$.
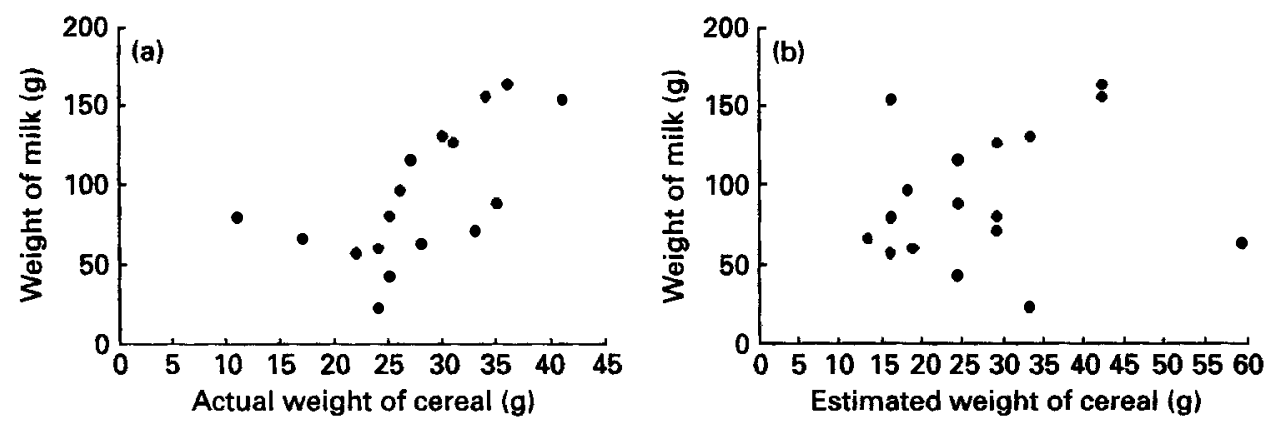

Fig. 3. Relationship between weight of milk (g) poured on to cereal and (a) actual weight of cereal and (b) weight of cereal estimated from photographs. For details of procedures, see pp. 33-36.

Table 8 shows the results from the sub-study on portion sizes of milk on cereal. Estimates of milk portion size using either quantitative descriptors (number of tablespoons, fractions of a pint or the bowl) or qualitative descriptors (small, medium large; damp, normal, drowned) were compared with actual weights consumed. There was a positive association $(r 0.52, P<0.05)$ between the amount of milk and the amount of cereal actually eaten (Fig. $3(\mathrm{a}))$, which fell to $r 0.10(P>0.05)$ in relation to estimates of portion size of cereal based on photographs (Fig. $3(\mathrm{~b})$ ). The qualitative descriptors were on average better related to the amounts of milk consumed than the quantitative descriptors, but there was substantial variation in the amount for any one term, and considerable overlap in amounts between terms (Fig. 4).

\section{DISCUSSION}

Subjects were selected from a wide variety of ages, social backgrounds and occupations. They formed a typical cross-section of the local population. While it cannot be said that the sample was representative of the population generally, it seems unlikely that subjects 


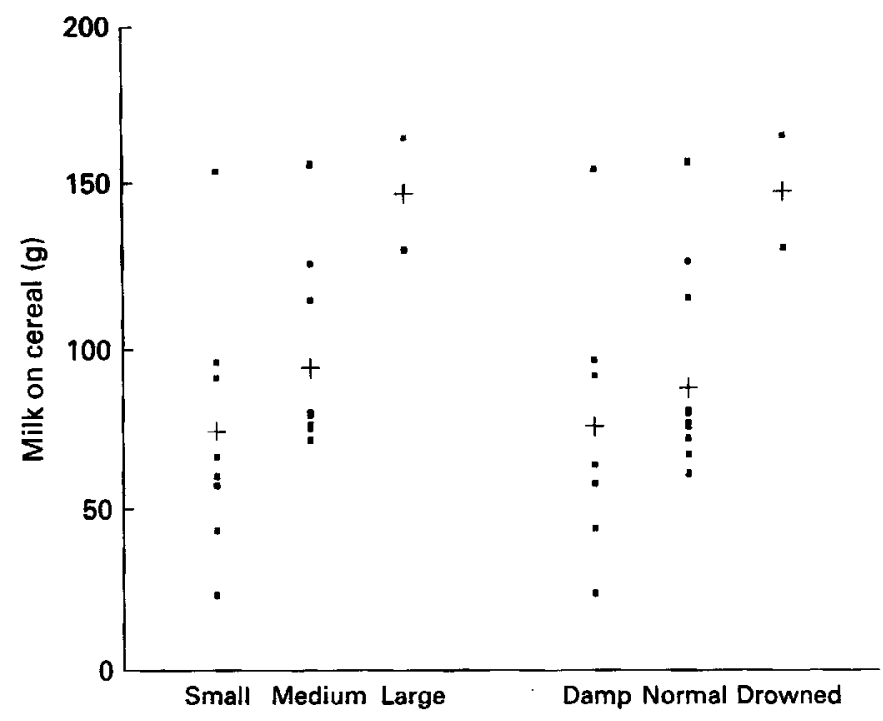

Fig. 4. Actual weights (g) of milk poured on to cereal by subjects who were subsequently asked to assess the portion size they used with various descriptors. For details of procedures, see p. $34(+)$, Mean value.

Table 9. Average under- or overestimation of food portion size using photographs (as a percentage of the actual portion size), by sex, according to portion size, for six foods (boiled potato, mashed potato, quiche, cornflakes, spaghetti, and sliced meat) observed in stage 1 (the previous study on errors related to perception alone (Nelson et al. 1994)), and in stage 2 (the present study on errors related to conceptualization plus perception)*

(Mean values and standard deviations)

\begin{tabular}{|c|c|c|c|c|c|c|c|c|}
\hline & \multicolumn{3}{|c|}{ Males } & \multicolumn{3}{|c|}{ Females } & \multirow{2}{*}{\multicolumn{2}{|c|}{$\begin{array}{c}\text { ANOVA } \\
P \text { values for: }\end{array}$}} \\
\hline & \multirow{2}{*}{\multicolumn{2}{|c|}{$\begin{array}{c}\text { Difference } \\
(\%)\end{array}$}} & \multirow[b]{2}{*}{ SD } & \multicolumn{3}{|c|}{ Difference } & & \\
\hline & & & & $n$ & $(\%)$ & SD & Stage & Sex \\
\hline \multicolumn{9}{|c|}{$\begin{array}{l}\text { Portion size consumed } \\
\text { Small }\end{array}$} \\
\hline Stage 1 & 563 & $2 \cdot 3$ & $36 \cdot 9$ & 640 & $5 \cdot 5$ & $42 \cdot 2$ & \multirow[t]{2}{*}{$0 \cdot 028$} & \multirow[t]{2}{*}{0.278} \\
\hline Stage 2 & 14 & $31 \cdot 6$ & 33.7 & 32 & $11 \cdot 6$ & $47 \cdot 1$ & & \\
\hline \multicolumn{9}{|l|}{ Medium } \\
\hline Stage 1 & 564 & -0.3 & $22 \cdot 6$ & 639 & $5 \cdot 9$ & $21 \cdot 8$ & \multirow[t]{2}{*}{$0 \cdot 047$} & \multirow[t]{2}{*}{0.000} \\
\hline Stage 2 & 33 & $-5 \cdot 9$ & $24 \cdot 7$ & 25 & -0.6 & $30 \cdot 2$ & & \\
\hline \multicolumn{9}{|l|}{ Large } \\
\hline Stage 1 & 573 & $-6 \cdot 2$ & $17 \cdot 4$ & 646 & $-2 \cdot 9$ & $13 \cdot 9$ & \multirow[t]{2}{*}{0.069} & \multirow[t]{2}{*}{0.000} \\
\hline Stage 2 & 8 & $-15 \cdot 3$ & $18 \cdot 6$ & 2 & $-11 \cdot 9$ & 13.0 & & \\
\hline \multicolumn{9}{|c|}{ All portion sizes } \\
\hline Stage 1 & 1700 & -1.4 & $27 \cdot 1$ & 1925 & $2 \cdot 8$ & 36.8 & \multirow[t]{2}{*}{$0 \cdot 229$} & \multirow[t]{2}{*}{0.000} \\
\hline Stage 2 & 55 & $2 \cdot 3$ & $31 \cdot 4$ & 59 & $5 \cdot 6$ & $40 \cdot 1$ & & \\
\hline
\end{tabular}

* For details of subjects and procedures in the present study, see Table 1 and pp. 33-36.

living in London would differ in their perception of food-portion size from subjects living elsewhere in Britain.

The wide variation in the percentage of over- or underestimation of portion size shown in Table 2 was initially thought likely to be related to the proportion of elderly subjects eating each food. In practice this was not observed. For example, foods such as chips and 
broccoli were overestimated, although none of the elderly subjects consumed these foods. Part of the wide range in variation in mean over- or underestimate may be attributed to the small number of observations for each food. The very substantial overestimation of butter and margarine was due to the methods of presentation of quantities in the photographs as increasing blocks of butter from 3 to $29 \mathrm{~g}$. There were too few representations of very small quantities, and $29 \mathrm{~g}$ was too large as the upper limit of portion size. An alternative means of representing these foods needs to be adopted, possibly by showing amounts actually spread on bread and crackers together with the amount spread shown on the end of a knife. The reason for the slope greater than unity for the shepherd's pie regression analysis is not clear.

The very large overestimation of small portion size (Table 3) was attributable in part to butter and margarine. Even when these foods were omitted from the analysis, however, the overestimation of small portion sizes persisted. This, together with the underestimation of large portion sizes, constitutes a true 'regression to the mean' effect.

The errors related to conceptualization plus perception were greater than those related to perception alone. Table 9 compares the results from the previous study (stage 1 ; Nelson et al. 1994) with those from the present study (stage 2) for the six foods evaluated in both studies. The variances (SD) of the percentage differences for the different portion sizes were similar in both stages. The means, however, differed significantly between stage 1 and stage 2 for small and medium portion sizes when controlling for sex in an ANOVA, and between sexes for medium and large portion sizes when controlling for stage. The results suggest that in both men and women there was a marked tendency to overestimate small portion sizes (especially in men) and to underestimate large portion sizes to a greater extent when relying on conceptualization skills (and having consumed the food) than when using perceptual skills alone. For example, the average overestimation of small portion sizes by males was $2.3 \%$ in stage 1 but $31.6 \%$ in stage 2 , suggesting that errors related to conceptualization contribute far more than errors in perception to the average error in the estimation of portion size. In females the difference between the error in stage $1(5.5 \%)$ and the error in stage $2(11.6 \%)$ suggests that conceptualization contributes an additional $6 \%$ to the average error in the overestimate of small portion sizes, over and above that contributed by perception alone. In spite of the large difference in errors between stages in men, the differences were statistically significant only in relation to stage (two-factor ANOVA, $P=0.028)$ and not between sexes $(P=0.278)$. There were no significant interactions between sex and stage.

In relation to the medium portions, errors in conceptualization resulted in an estimate of portion size on average $6 \%$ less than the estimate based on perception alone (for men: $-5.9-(-0.3)=-5.6$; for women: $-0.6-5.9=-6.5)$. Differences were statistically significant between stage $(P=0.047)$ and $\operatorname{sex}(P=0.000)$ (two-factor ANOVA) and again there were no interactions between stage and sex. Errors in the conceptualization of the large portion sizes resulted in average underestimates of portion size about $9 \%$ below those related to perception alone (for men: $-15 \cdot 3-(-6 \cdot 2)=-9 \cdot 1$; for women, $-11 \cdot 9-(-2 \cdot 9)=-9 \cdot 0)$.

Conceptualization thus appears to compound the error related to perception alone, and to accentuate the 'regression to the mean' effect. This important effect was obscured when all portion sizes were assessed together. The differences in the errors between stage 1 and stage 2 appeared to be negligible and were not statistically significant $(P=0.229$, twofactor ANOVA with stage and sex as main factors), as the errors in the estimates relating to the small and the large portion sizes cancelled each other out.

These findings regarding differential errors in the conceptualization of portion size imply that subjects consuming small portions are more likely to be misclassified according to their 
estimates of food consumption than those eating medium or large portions. Between 70 and $75 \%$ of medium or large portion sizes were estimated correctly to within $\pm 30 \%$ of actual portion size, but this fell to $50 \%$ for small portion sizes. Part of this effect is related to the smaller denominators used to calculate the percentage error. However, misclassification of subjects according to level of food consumption within groups of subjects who habitually eat smaller portions (e.g. women, children, older subjects) will be greater than that in groups who habitually eat larger portions (e.g. men, younger subjects). The differential errors according to age (Table 3 ) imply that subjects aged 45 years and over are more likely to be misclassified according to their food consumption than younger subjects, due in part to their greater consumption of small portions, and in part to an independent 'age' effect.

The errors relating to estimates of portion size are reflected in the estimates of the nutrient content of the meals to which these foods contributed. Thus, with the exception of vitamin $\mathrm{C}$ (and excluding butter and margarine from the calculation), the average errors were within $\pm 7 \%$ of the calculated energy and nutrient contents of the meals based on actual portion sizes. Errors of this size may be acceptable when estimating the nutrient intake of groups of people. Individuals, however, varied considerably in the extent to which the calculated nutrient content of meals reflected the actual nutrient content (Fig. 2). This variation was pronounced between different age and BMI groups (Table 5), although the latter was of borderline statistical significance for energy $(P=0.069)$. The error relating to vitamin $C$ reflects the tendency on the part of a few individuals to overestimate the portion sizes of chips, peas, and boiled cabbage. This type of error will lead to the misclassification of a subset of individuals (not readily identified) who overestimate the consumption of foods which are rich in one particular nutrient.

In surveys which include subjects from a wide range of ages and/or body sizes the use of photographs to estimate food consumption and nutrient intake may introduce errors of misclassification which need to be addressed in the course of analysis. It is clear from Table 6 that misclassification is reduced when photographs are used in preference to average portion sizes. Further adjustment for differential errors in the estimation of portion size may be possible using appropriate statistical techniques to improve estimates of the strength of diet-disease associations (Nelson, 1991).

Lastly, it is important to acknowledge the difficulty in estimating amounts of milk served on cereal. Correlations between the amount of milk and the quantity of cereal were weak, particularly when the quantity of cereal was estimated from photographs (Fig. 3). The number of tablespoons was a poor measure. Fractions of a pint or portion of the bowl differentiated the highest and lowest amounts, but the middle range of measures were poorly distinguished. Qualitative descriptors provided more consistent average measures, which is perhaps not surprising because there were fewer of them, but the overlap between categories was substantial (Fig. 4). The best separation was obtained using the simple terms 'small', 'medium' and 'large' to indicate the size of the serving of milk. For groups of subjects this will probably be satisfactory, but for individuals substantial misclassification will result, and will be reflected in estimates of $\mathrm{Ca}$ especially.

We confirm the conclusion from our previous paper (Nelson et al. 1994) that photographs are of benefit in estimating food-portion size. The evidence from the present study shows clearly that compared with average portion sizes, photographs improve estimates of the nutrient content of meals and reduce subject misclassification. We will shortly be reporting the effects of using photographs on estimates of nutrient intake over $24 \mathrm{~h}$, and comparing the use of four $v$. eight photographs.

The authors would like to thank James Meyer for the photography, the Ministry of Agriculture, Fisheries and Food for the grant to undertake the work, members of the 
Nutritional Epidemiology Group Steering Committee who oversaw the design of the project and commented constructively on its analysis, and the volunteers for taking part in the study. Membership of the Nutritional Epidemiology Group Steering Committee: Ms Mary Atkinson (KCL), Miss Alison Black (MRC Dunn Nutrition Unit), Dr Joyce Hughes (MAFF), Mr James Meyer, Ms Alison Mills (MAFF), Dr Michael Nelson (KCL), Dr Richard Shepherd (AFRC Institute of Food Research), Dr Margaret Thorogood (London School of Hygiene and Tropical Medicine). Corresponding members: Dr Annie Anderson (University of Glasgow), Ms Mary Cooper (St Mary's Hospital, Leeds), Dr Barbara Livingstone (Ulster).

\section{REFERENCES}

Byers, T., Marshall, J., Fiedler, R., Zielezny, M. \& Graham, S. (1985). Assessing nutrient intake with an abbreviated dietary interview. American Journal of Epidemiology 122, 41-49.

Chu, S. Y., Kolonel, L. N., Hankin, J. H. \& Lee, J. (1984). A comparison of frequency and quantitative dietary methods for epidemiologic studies of diet and disease. American Journal of Epidemiology 119, 323-333.

Edington, J., Thorogood, M., Geekie, M., Ball, M. \& Mann, J. (1989). Assessment of nutritional intake using dietary records with estimated weights. Journal of Human Nutrition and Dietetics 2, 407-414.

Faggiano, F., Vineis, P., Cravanzola, D., Pisani, P., Xompero, G., Riboli, E. \& Kaaks, R. (1992). Validation of a method for the estimation of food portion size. Epidemiology 3, 379-382.

Gaskell, G., Wright, D. \& O'Muircheartaigh, C. (1993). Reliability of surveys. Psychologist November, 500-503.

Gregory, J., Foster, K., Tyler, H. \& Wiseman, M. (1990). The Dietary and Nutritional Survey of British Adults. London: H.M. Stationery Office.

Guthrie, H. A. (1984). Selection and quantification of typical food portions by young adults. Journal of the American Dietetic Association 12, 1440-1444.

Hankin, J. H., Wilkins, L. R., Kolonel, L. N. \& Yoshizawa, C. N. (1991). Validation of a quantitative diet history method in Hawaii. American Journal of Epidemiology 133, 616-628.

Haraldsdottir, J., Tjønneland, A. \& Overvad, K. (1994). Validity of individual portion size estimates in a food frequency questionnaire. International Journal of Epidemiology 23, 787-796.

Holland, B., Unwin, I. D. \& Buss, D. H. (1988). Cereals and Cereal Products. Third Supplement to McCance and Widdowson's The Composition of Foods, 4th ed. Cambridge: Royal Society of Chemistry.

Holland, B., Unwin, I. D. \& Buss, D. H. (1989). Milk Products and Eggs. Fourth Supplement to McCance and Widdowson's The Composition of Foods, 4th ed. Cambridge: Royal Society of Chemistry.

Holland, B., Unwin, I. D. \& Buss, D. H. (1991 a). Vegetables, Herbs and Spices. Fifth Supplement to McCance and Widdowson's The Composition of Foods, 4th ed. Cambridge: Royal Society of Chemistry.

Holland, B., Unwin, I. D. \& Buss, D. H. (1992a). Fruit and Nuts. First Supplement to McCance and Widdowson's The Composition of Foods, 5th ed. Cambridge: Royal Society of Chemistry.

Holland, B., Welch, A. A. \& Buss, D. H. (1992b). Vegetable Dishes. Second Supplement to McCance and Widdowson's The Composition of Foods, 5th ed. Cambridge: Royal Society of Chemistry.

Holland, B., Welch, A. A., Unwin, I. D., Buss, D. H., Paul, A. A. \& Southgate, D. A. T. (1991 b). McCance and Widdowson's The Composition of Foods, 5th ed. Cambridge: Royal Society of Chemistry.

Lee, P. \& Cunningham, K. (1990). Irish National Nutrition Survey. Dublin: Irish Nutrition and Dietetics Institute.

Morgan, S., Flint, D. M., Prinsley, M. L., Wahlqvist, M. L. \& Parish, A. E. (1982). Measurement of food intake in the elderly by food photography. Proceedings of the Nutrition Society of Australia 7, 172.

Nelson, M. (1991). The validation of dietary questionnaires. In Design Concepts in Nutritional Epidemiology, pp. 266-296 [B. M. Margetts and M. Nelson, editors]. Oxford: Oxford University Press.

Nelson, M., Atkinson, M. \& Darbyshire, S. (1994). Food photography I: the perception of food portion size from photographs. British Journal of Nutrition 72, 649-663.

Pietinen, P., Hartman, A. M., Haapa, E., Rasanen, L., Haapakoski, J., Palmgren, J., Albanes, D., Virtamo, J. \& Huttunen, J. K. $(1988 a)$. Reproducibility and validity of dietary assessment instruments. I. A self-administered food use questionnaire with a portion size picture booklet. American Journal of Epidemiology 128, 655-666.

Pietinen, P., Hartman, A. M., Haapa, E., Rasanen, L., Haapakoski, J., Palmgren, J., Albanes, D., Virtamo, J. \& Huttunen, J. K. (1988b). Reproducibility and validity of dietary assessment instruments. II. A qualitative food frequency questionnaire. American Journal of Epidemiology 128, 667-675.

Rutishauser, I. H. E. (1982). Food models, photographs or household measures? Proceedings of the Nutrition Society of Australia 7, 144-145.

Samet, J. M., Humble, C. G. \& Skipper, B. E. (1984). Alternatives in the collection and analysis of food frequency interview data. American Journal of Epidemiology 120, 572-581.

Tan, S. P., Wenlock, R. W. \& Buss, D. H. (1985). Immigrant Foods. Second Supplement to McCance and Widdowson's The Composition of Foods, 4th ed. London: H.M. Stationery Office. 
Tjønneland, A., Overvad, K., Haraldsdottir, J., Bang, S., Ewertz, M. \& Jenson, O. M. (1991). Validation of a semi-quantitative food frequency questionnaire developed in Denmark. International Journal of Epidemiology 20, $906-912$.

\section{APPENDIX}

Menus offered to subjects in study

Subjects were asked to confirm at the beginning of the study that they would be willing to eat any of the foods listed. When a subject arrived at the College for a meal, they were asked to help themselves to each of the foods on offer. Subjects were mainly cooperative by taking all foods from the menu offered.

The food combinations specified for each meal as far as possible resembled realistic choices to allow for a genuine reflection of serving sizes usually chosen by subjects. The original protocol called for each menu to be served to ten subjects, giving 140 subjects in total. In practice we achieved 136.

Each meat and carbohydrate dish was included in two different menus, whereas each vegetable and each dessert was present in three, with the exception of baked beans which were included in both breakfast menus as baked beans on toast. Butter or margarine was included twice for spreading on toast (breakfast), three times for spreading on biscuits or crackers (dessert), and eleven times as an addition to vegetables. Cheese was included three times as a wedge, chunk or slice with biscuits as a dessert, and twice grated as an accompaniment to spaghetti bolognese.

The menus served were as follows:

1. Roast beef

Mashed potato

Peas

Cheddar cheese (and biscuits)

Butter/margarine (for cheese, and for mashed potato)

2. Roast beef

Boiled potato

Boiled cabbage

Butter/margarine (with potato, cabbage)

Ice-cream

3. Beef stew

Boiled rice

Broccoli

Butter/margarine (with broccoli)

Ice-cream

4. Beef stew

Mashed potato

Baked beans

Cheddar cheese (and biscuits)

Butter/margarine (with cheese and mashed potato)

5. Shepherd's pie

Baked beans

Rice pudding

6. Shepherd's pie

Peas

Sponge cake 
7. Bolognese sauce

Boiled spaghetti

Grated cheddar cheese (with bolognese sauce)

Cabbage

Butter/margarine (with cabbage)

Sponge cake

8. Bolognese sauce

Boiled spaghetti

Grated cheddar cheese (with bolognese sauce)

Broccoli

Rice pudding

Butter/margarine (with broccoli)

9. Fillet/sirloin steak

Chips

Peas

Butter/margarine (with peas)

Sponge cake

10. Fillet/sirloin steak

Rice

Cabbage

Cheese (with biscuits)

Butter/margarine (with broccoli and biscuits)

11. Bacon-and-egg quiche

Boiled potatoes

Broccoli

Butter/margarine (with potatoes, cabbage)

Ice-cream

12. Bacon-and-egg quiche

Chips

Baked beans

Rice pudding

13. Cornflakes

Milk

Butter/margarine (with toast + spread)

Baked beans (with buttered toast)

14. Cornflakes

Milk

Butter/margarine (with toast + spread)

Baked beans (with buttered toast) 